\title{
Case Report: Typhoid Fever Complicated by lleal Perforation in an Urban Slum of Dhaka, Bangladesh
}

\author{
Farhana Khanam, ${ }^{1,2}$ Thomas C. Darton, ${ }^{3}$ Allen G. P. Ross, ${ }^{1}$ K. Zaman, ${ }^{1}$ Andrew J. Pollard, ${ }^{4}$ John D. Clemens, ${ }^{1}$ and Firdausi Qadri ${ }^{1 *}$ \\ ${ }^{1}$ icddr,b, (International Centre for Diarrhoeal Disease Research, Bangladesh), Dhaka, Bangladesh; ${ }^{2}$ School of Medical Science, Griffith University, \\ Gold Coast, Australia; ${ }^{3}$ Department of Infection, Immunity and Cardiovascular Disease, The Florey Institute for Host-Pathogen Interactions, \\ University of Sheffield, Sheffield, United Kingdom; ${ }^{4}$ Oxford Vaccine Group, Department of Paediatrics, NIHR Oxford Biomedical Research Centre, \\ University of Oxford, Oxford, United Kingdom
}

\begin{abstract}
Intestinal perforation is one of the most dangerous complications of typhoid fever and demands urgent hospitalization, diagnosis, and surgical management to reduce morbidity and prevent mortality. Here, we report a case of typhoidal intestinal perforation in a 19 year-old young man detected by passive surveillance during a cluster-randomized trial with Vi-tetanus toxoid conjugate vaccine (Typhoid Vaccine Acceleration Consortium: TyVAC) in an urban slum area in Mirpur, Dhaka, Bangladesh. The patient presented with a high-grade fever, lower abdominal pain, and vomiting and was admitted to a healthcare facility. Physical examination and preoperative investigations of the patient suggested a presumptive diagnosis of intestinal perforation, and the patient was transferred to a tertiary-level hospital for surgical management. A positive blood culture, intraoperative findings, and histopathology of an intestinal biopsy confirmed ileal perforation due to typhoid fever. This case report highlights the need for prompt diagnosis and appropriate pre- and postoperative management of patients who appear with the symptoms of typhoidal intestinal perforation. This report further demonstrates the importance of systematic surveillance and proper evaluation to determine the true incidence rate of typhoid fever and intestinal perforation in Bangladesh.
\end{abstract}

\section{INTRODUCTION}

lleal perforation is one of the most fatal and common complications of typhoid fever, ${ }^{1}$ which is a public health concern in many low-resource settings. ${ }^{2}$ Estimates of typhoidal intestinal perforation (TIP) incidence in developed and resource-poor countries vary widely from $0.8 \%$ to $39 \%,{ }^{3-6}$ likely because of 1 ) nonstandardized case definitions, 2) poor reporting or surveillance systems, and 3) lack of clinician awareness. Several risk factors have been found to be associated with ileal perforation, including inappropriate antibiotic therapy and male gender. ${ }^{7}$ Despite recent significant improvement in how cases of TIP are managed, the outcome of patients is often poor. With an associated case fatality rate (CFR) of 5-75\% globally, ${ }^{4,8,9}$ intestinal perforation remains the most frequent cause of death in patients with typhoid fever in endemic areas. ${ }^{6}$ The median CFR among surgical studies of typhoid fever patients was $15.5 \%$ (6.7-24.1\%), reported by a systematic review and meta-analysis on complication and CFR of the disease. ${ }^{10}$ Surgical factors associated with CFRs include ileostomy technique, duration of the preoperative period, and peritoneal contamination. Age, number, and location of perforations are also found to influence the CFR. ${ }^{4,8}$

Bangladesh is an endemic zone for typhoid fever, ${ }^{11}$ although few cases of TIP have previously been reported. ${ }^{6} \mathrm{~A}$ systematic passive surveillance program for enteric fever (including typhoid and paratyphoid) as part of the Strategic Typhoid alliance across Africa and Asia project was carried out in an urban slum area, Mirpur, Dhaka city in Bangladesh. ${ }^{12}$ High incidence rates of both typhoid and paratyphoid fever were found in this febrile group (manuscript submitted). A clusterrandomized trial with Vi-tetanus TCV (Vi-TCV) is also being conducted in Mirpur slum area under the Typhoid Vaccine

*Address correspondence to Firdausi Qadri, Infectious Diseases Division, International Centre for Diarrhoeal Disease Research, Bangladesh, 68 Shaheed Tajuddin Ahmed Sharani, Dhaka 1212, Bangladesh. E-mail: fqadri@icddrb.org
Acceleration Consortium, ${ }^{13}$ where one patient presented with fever and intestinal perforation in passive surveillance of the vaccine study. We report the case of intestinal perforation due to typhoid fever here.

\section{CASE DESCRIPTION}

A 19-year-old male patient was admitted to one of the healthcare facilities conducting passive surveillance as part of the TyVAC project, with symptoms of high-grade fever $\left(\geq 38.8^{\circ} \mathrm{C}\right)$ for 6 days accompanied by severe lower abdominal pain and vomiting. Three days before admission, the patient had started self-administering cefixime, a third-generation cephalosporin antibiotic obtained without prescription from a local pharmacy. On physical examination following admission, the patient's axillary temperature was $38.5^{\circ} \mathrm{C}$, and he was toxic and lethargic but without signs of dehydration. Abdominal examination demonstrated rebound tenderness and absence of bowel sounds on auscultation. An upright abdominal radiograph (Figure 1) demonstrated multiple air fluid levels in a step-ladder pattern with free gas evident under both domes of the diaphragm. A diagnosis of intestinal perforation was made, and the patient was resuscitated with intravenous solution and antispasmodic and antiulcer medication and kept nil by mouth. Broad-spectrum intravenous antibiotics (thirdgeneration cephalosporin and ceftriaxone) and metronidazole were initiated. With consent, blood was collected for culture and sensitivity and hematological and biochemical investigations. The patient was then referred to a tertiary hospital for definitive surgical management, which included a laparotomy and ileal perforation repair with ileostomy.

Blood culture was carried out using a standard automated BacT/ALERT method in the icddr,b laboratory, and Gramnegative bacilli were grown within 8-10 hours, further identified as Salmonella enterica serovar Typhi from subculture based on colony morphology, biochemical tests, and slide agglutination tests using Salmonella-specific antisera (Denka Sieken, Tokyo, Japan). ${ }^{14}$ Antimicrobial susceptibility testing was 


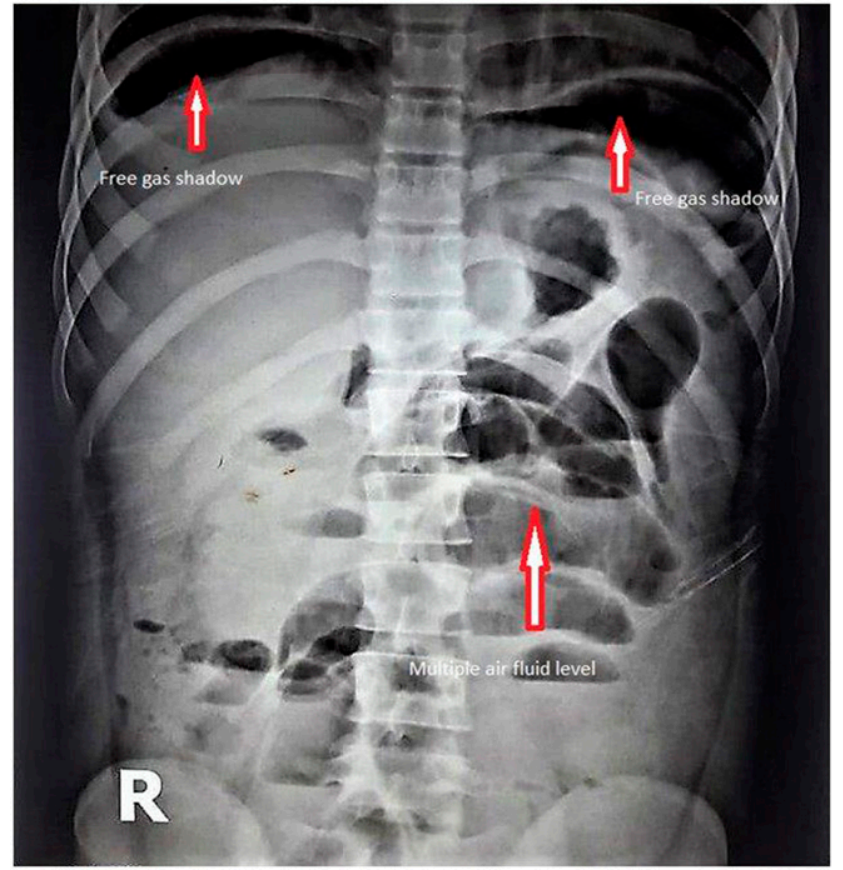

FIGURE 1. Plain abdominal radiograph. This figure appears in color at www.ajtmh.org.

carried out by the disk diffusion method following the CLSI guidelines ${ }^{15}$ and demonstrated sensitivity to chloramphenicol, co-trimoxazole, cefixime, ceftriaxone, and meropenem; resistance to ampicillin, azithromycin, ciprofloxacin, and nalidixic acid; and intermediate sensitivity to amoxiclav. Other laboratory investigations showed $33.3 \%$ hematocrit, $13,500 / \mathrm{cm}^{3}$ white blood cell count, $475,000 / \mathrm{cm}^{3}$ platelets, $140 \mathrm{mmol} / \mathrm{L}$ sodium, 98 $\mathrm{mmol} / \mathrm{L}$ chloride, $3.77 \mathrm{mmol} / \mathrm{L}$ potassium, $0.79 \mathrm{mg} / \mathrm{dL}$ creatinine, $18 \mathrm{U} / \mathrm{L}$ alanine aminotransferase, and $9.53 \mu \mathrm{g} / \mathrm{mL}$ D-dimer.

During laparotomy, approximately $1,400 \mathrm{~mL}$ of pus was removed from the abdomen, and the gut was found to be edematous. Ceftriaxone therapy was replaced with intravenous meropenem. All intravenous antibiotics were continued until postoperative day 5 , and then, oral antibiotic therapy was initiated. Other intraoperative findings included a perforation of $3 \times 4 \mathrm{~cm}$ in diameter which was $10 \mathrm{~cm}$ proximal to the ileocecal junction. Histopathological examination of the resected intestinal tissue revealed an ulcer lined by fibrinopurulent exudate supported by granulation tissue, with associated edema of the submucosa and serosal infiltration by acute and chronic inflammatory cells. There were no granulomatous or malignant changes found in the tissue specimens obtained. Pus collected from the abdomen during operation was cultured for bacteria but yielded no growth.

On postoperative day 3 , the surgical wound was found to be infected and wound dehiscence subsequently developed. A swab from the operation wound cultured Klebsiella spp., which was interpreted to be colonizing flora only. Wound dressing and antibiotic therapy were continued until postoperative day 23 when secondary closure of wound dehiscence was performed under local anesthesia. The patient was discharged on postoperative day 26 , with a further 7 -day course of oral linezolid.

The patient was hospitalized again after 3 months of the original ileostomy operation for carrying out the reversal operation. The operation was carried out under general anesthesia, and the patient was followed up until recovery.

\section{DISCUSSION}

Intestinal perforation is a devastating complication of typhoid fever and the most common cause of death. The mechanism whereby infection results in perforation is due to bacterial attachment and invasion, causing subsequent hyperplasia and necrosis of the immune sentinel microfold cells, anatomically visible in Peyer's patches. ${ }^{6}$ The usual site of perforation is at the anti-mesenteric border of the ileum; however, other sites can also be affected such as the cecum, jejunum, colon, ${ }^{1}$ and, occasionally, the appendix. ${ }^{16}$ This report describes a case of ileal perforation due to S. Typhi infection detected during passive surveillance at our field site in Dhaka, Bangladesh. Because of consumption of contaminated food and water outside home, middle-aged/adult men are mostly affected with TIP, ${ }^{6}$ as seen in the case of our patient who was a 19-year-old young man with a history of consuming foods obtained from outside the home setting. In addition, the patient had been residing in one of the urban slums of Dhaka city of Bangladesh, where there are deficiencies in sewage treatment and waste disposal, which results in fecal contamination of the environment. During rainy season, open sewage that flows outside the doors of homes in slums enters into homes and contaminates domestic water source. These are important risk factors for transmitting S. Typhi infection in the community. ${ }^{17,18}$

Although TIP usually develops in the second or third week after the onset of symptoms, ${ }^{19,20}$ our patient developed the symptoms of TIP within 6 days of fever. Many reports from resource-limited settings have shown that symptoms of bowel perforation often develop within the first week of illness. ${ }^{21}$ Hypersensitivity of Peyer's patches and lower background immunity of patients, coupled with bacterial virulence factors such as antimicrobial resistance, have been suggested predisposing factors for early presentation with TIP. ${ }^{19}$ Case fatality rates are reported to be higher in cases where perforation developed after the third week of illness, ${ }^{22,23}$ whereas lower fatality rates occur with prompt surgical management in cases where symptoms of TIP appeared within the first week of illness. $^{5,22}$ Early appearance of symptoms, immediate diagnosis, and timely surgical intervention were the pivotal factors favoring a good prognosis of the patient in our report.

Diagnosis of presumptive TIP usually depends on clinical presentation, intraoperative findings, and other laboratory investigations including histopathological report of intestinal tissue. Blood cultures reveal the positive result for $S$. Typhi in only 3-34\% cases of TIP. ${ }^{24,25}$ A preliminary diagnosis of the patient in this report was made based on the preoperative physical examination and abdominal X-ray. Positive blood culture report for S. Typhi, observations made by the surgeon during operation, and histopathological examination of the intestinal biopsy confirmed the diagnosis. In addition, nontraumatic ileal perforation for visitors and residents in a typhoid-endemic country should be considered as TIP, suggested by the WHO VaccinePreventable Diseases Surveillance Standards. ${ }^{26}$

As with other types of abdominal surgery, wound infection is a common cause of postoperative morbidity in cases of intestinal perforation due to typhoid fever. ${ }^{1,19}$ In this case, postoperative wound infection and wound dehiscence were 
developed after the operation. The infection was managed by regular wound dressing and appropriate antibiotic therapy and secondary closer of the wound. The patient was discharged from the hospital with oral antibiotic therapy for additional 7 days.

This rare case highlights the underrecognized, often fatal, complication of intestinal perforation in typhoid fever. It was detected in this particular case because of the patient being a participant in a typhoid surveillance survey and the awareness of local clinicians. Because intestinal perforation is a common, but deadly, complication of typhoid fever in developing countries, ${ }^{27}$ systemic surveillance related to operations performed for intestinal perforation needs to be included to accurately estimate the typhoid-associated illness burden in endemic settings, like Bangladesh. In addition to accurate surveillance, prevention measures (e.g., boiling water and handwashing), typhoid conjugate vaccination, and prompt treatment in high-quality surgical facilities are vital to reduce this important and underestimated surgical presentation.

Received November 10, 2020. Accepted for publication January 11, 2021.

Published online March 22, 2021.

Acknowledgments: We are thankful to the Bill \& Melinda Gates Foundation (OPP1151153) for the support. The icddr,b is also grateful to the government of Bangladesh, Canada, Sweden, and the United Kingdom for providing core/unrestricted support.

Disclosure: AP is the Chair of UK Dept. Health and Social Care's (DHSC) Joint Committee on Vaccination \& Immunisation (JCVI), and is a member of the WHO's SAGE. The views expressed in this article do not necessarily represent the views of DHSC, JCVI, NIHR or WHO. The University of Oxford has entered into a partnership with AstraZeneca on coronavirus vaccine development.

Authors' addresses: Farhana Khanam, Infectious Diseases Division, icddr,b, (International Centre for Diarrhoeal Disease Research, Bangladesh), Dhaka, Bangladesh, and School of Medical Science, Griffith University, Gold Coast, Australia, E-mail: farhanak@icddrb.org. Thomas C. Darton, Department of Infection, Immunity and Cardiovascular Disease, Florey Institute for Host-Pathogen Interactions, University of Sheffield, Sheffield, United Kingdom, E-mail: t.darton@ sheffield.ac.uk. Allen G. P. Ross, K. Zaman, John D Clemens, and Firdausi Qadri, Infectious Diseases Division, icddr,b, (International Centre for Diarrhoeal Disease Research, Bangladesh), Dhaka, Bangladesh, E-mails: allen.ross@icddrb.org, kzaman@icddrb.org, jclemens@ icddrb.org, and fqadri@icddrb.org. Andrew J. Pollard, Oxford Vaccine Group, Department of Paediatrics, University of Oxford, Oxford, United Kingdom, E-mail: andrew.pollard@paediatrics.ox.ac.uk.

This is an open-access article distributed under the terms of the Creative Commons Attribution (CC-BY) License, which permits unrestricted use, distribution, and reproduction in any medium, provided the original author and source are credited.

\section{REFERENCES}

1. Pujar KA, Ashok AC, Rudresh HK, Srikantaiah HC, Girish KS, Suhas KR, 2013. Mortality in typhoid intestinal perforation-a declining trend. J Clin Diagn Res 7: 1946-1948.

2. Bhutta ZA, Gaffey MF, Crump JA, Steele D, Breiman RF, Mintz ED, Black RE, Luby SP, Levine MM, 2018. Typhoid fever: way forward. Am J Trop Med Hyg 99: 89-96.

3. Chiu CH, Tsai JR, Ou JT, Lin TY, 2000. Typhoid fever in children: a fourteen-year experience. Acta Paediatr Taiwan 41: 28-32.

4. Atamanalp SS, Aydinli B, Ozturk G, Oren D, Basoglu M, Yildirgan $\mathrm{MI}, 2007$. Typhoid intestinal perforations: twenty-six year experience. World J Surg 31: 1883-1888.

5. Eggleston FC, Santoshi B, Singh CM, 1979. Typhoid perforation of the bowel. Experiences in 78 cases. Ann Surgery 190: 31-35.
6. Butler T, Knight J, Nath SK, Speelman P, Roy SK, Azad MA, 1985. Typhoid fever complicated by intestinal perforation: a persisting fatal disease requiring surgical management. Rev Infect Dis 7: 244-256.

7. Hosoglu S, Aldemir M, Akalin S, Geyik MF, Tacyildiz IH, Loeb M, 2004. Risk factors for enteric perforation in patients with typhoid Fever. Am J Epidemiol 160: 46-50.

8. Santillana M, 1991. Surgical complications of typhoid fever: enteric perforation. World J Surg 15: 170-175.

9. Birkhold M, Coulibaly Y, Coulibaly O, Dembélé P, Kim DS, Sow S, Neuzil KM, 2020. Morbidity and mortality of typhoid intestinal perforation among children in sub-Saharan Africa 1995-2019: a scoping review. World J Surg 44: 2892-2902.

10. Marchello CS, Birkhold M, Crump JA, 2020. Complications and mortality of typhoid fever: a global systematic review and metaanalysis. J Infect 81: 902-910.

11. Saha SK, Baqui $A H$, Hanif $M$, Darmstadt GL, Ruhulamin $M$, Nagatake T, Santosham M, Black RE, 2001. Typhoid fever in Bangladesh: implications for vaccination policy. Pediatr Infect Dis J 20: 521-524.

12. Darton TC et al., 2017. The STRATAA study protocol: a programme to assess the burden of enteric fever in Bangladesh, Malawi and Nepal using prospective population census, passive surveillance, serological studies and healthcare utilisation surveys. BMJ Open 7: e016283.

13. Theiss-Nyland $\mathrm{K}$ et al., 2019. Assessing the impact of a vipolysaccharide conjugate vaccine in preventing typhoid infection among Bangladeshi children: a protocol for a phase Illb trial. Clin Infect Dis 68: S74-S82.

14. Sheikh A et al., 2009. Salmonella enterica serovar Typhi-specific immunoglobulin A antibody responses in plasma and antibody in lymphocyte supernatant specimens in Bangladeshi patients with suspected typhoid fever. Clin Vaccin Immunol 16: 1587-1594.

15. CLSI, 2019. Performance Standards for Antimicrobial Susceptibility Testing, 29th edition. CLSI supplement M100. Wayne, PA: Clinical and Laboratory Standards Institute.

16. Golakai VK, Makunike R, 1997. Perforation of terminal ileum and appendix in typhoid enteritis: report of two cases. East Afr Med J 74: 796-799.

17. Amin $\mathrm{N}$ et al., 2019. Quantitative assessment of fecal contamination in multiple environmental sample types in urban communities in Dhaka, Bangladesh using SaniPath microbial approach. PLoS One 14: e0221193.

18. Dewan AM, Corner R, Hashizume M, Ongee ET, 2013. Typhoid Fever and its association with environmental factors in the Dhaka Metropolitan Area of Bangladesh: a spatial and timeseries approach. PLos Negl Trop Dis 7: e1998.

19. Chalya PL, Mabula JB, Koy M, Kataraihya JB, Jaka H, Mshana SE, Mirambo M, Mchembe MD, Giiti G, Gilyoma JM, 2012. Typhoid intestinal perforations at a University teaching hospital in Northwestern Tanzania: a surgical experience of 104 cases in a resource-limited setting. World J Emerg Surg 7: 4.

20. Sumer A, Kemik O, Dulger AC, Olmez A, Hasirci I, Kişli E, Bayrak V, Bulut G, Kotan C, 2010. Outcome of surgical treatment of intestinal perforation in typhoid fever. World J Gastroenterol 6: 4164-4168.

21. Uba AF, Chirdan LB, Ituen AM, Mohammed AM, 2007. Typhoid intestinal perforation in children: a continuing scourge in a developing country. Pediatr Surg Int 23: 33-39.

22. Singh J, Singh B, 1975. Enteric perforation in typhoid fever: a study of 15 cases. Aust N Z J Surg 45: 279-284.

23. Dickson JA, Cole GJ, 1964. Perforation of the terminal ileum. A review of 38 cases. Br J Surg 51: 893-897.

24. Sharma AK, Sharma SK, Sharma A, Soni D, 2013. Typhoid intestinal perforation: 24 perforations in one patient. Ann Med Health Sci Res 3: S41-S43.

25. Keenan JP, Hadley GP, 1984. The surgical management of typhoid perforation in children. Br J Surg 71: 928-929.

26. World Health Organization, 2018. Vaccine-Preventable Diseases Surveillance Standards: Typhoid and Other Invasive Salmonellosis. Geneva, Switzerland: WHO, 1-13. Available at: https://www.who.int/ immunization/monitoring_surveillance/burden/vpd/WHO_ SurveillanceVaccinePreventable_21_Typhoid_BW_R1.pdf?ua=1.

27. Contini S, 2017. Typhoid intestinal perforation in developing countries: still unavoidable deaths? World J Gastroenterol 23: 1925-1931. 